\title{
RENAL ONTOGENY OF P-GLYCOPROTEIN/MDR1 IN RAT
}

\author{
Hodorova I. ${ }^{1}$, Mihalik J. ${ }^{1}$, Vecanova J. ${ }^{1}$, Dankova M. ${ }^{2}$, Rybarova S. ${ }^{1}$
}

${ }^{1}$ Department of Anatomy, P. J. Safarik University, Faculty of Medicine, Kosice, Slovak Republic; ${ }^{2}$ Department of Histology and Embryology, P. J. Safarik University, Faculty of Medicine, Kosice, Slovak Republic

\begin{abstract}
BACKGROUND: P-glycoprotein (Pgp/MDR1) is an ATP-dependent, integral plasma-membrane efflux pump that is constitutively expressed on adult apical brush-border epithelium of renal proximal tubules. This Pgp/ MDR1 tissue distribution and localization affects the absorption, distribution, metabolism, and excretion of Pgp/MDR1 substrates. The ontogeny of rat Pgp/MDR1 is still doubtful, and such knowledge may be helpful in understanding age-related pharmacokinetics. The purpose of this study was to determine, whether Pgp/ MDR1 expression is altered during development.

METHODS: Postnatal expression of Pgp was determined using immunohistochemical method. Tissue from Wistar rat were isolated on the 1 st day (D1), 7th day (D7), 14th day (D14), 21st day of life (D21) and from adult animals (60 days old; Ad).

RESULTS: Our ontogeny study illustrated that expression of Pgp was relatively constant from birth to adulthood. CONCLUSIONS: Knowledge of the ontogeny of transport proteins involved in distribution and elimination of drugs is important for adequate interpretation of the results of toxicity studies in juvenile animals.
\end{abstract}

Key words: Pgp/MDR1, rat, immunohistochemistry

\section{INTRODUCTION}

ATP-binding cassette $(\mathrm{ABC})$ superfamily now includes about 300 proteins, among the transporters of quite different compounds $(1,2,3)$. Proteins of this family are present in all living organisms. About fifty $\mathrm{ABC}$ proteins have been found in man and approximately the same number in mouse (4). Since a great number of ABC proteins were discovered only recently, many of them are still poorly studied. Studies of ABC proteins are important both for medicine and biology because they concern problems of protection of all living cells. Human proteins of the ABC family are divided to seven subfamilies (class A to G) $(4,5)$. The affiliation of each protein to a subfamily is determined by its domain organization, namely by the number and combination of transmembrane domains (TMDs) and ATP-binding domains (NBDs, nucleotide-binding domains) (6). In total, there are 49 protein members in this family, but only three are well known for their multidrug resistance (7). There are P-glycoprotein (Pgp/MDR1, ABCB1), MDR-associated protein (MRP1, $\mathrm{ABCC} 1$ ) and breast cancer resistance protein (BCRP, ABCG2). Pgp, paradigm ABC drug efflux transporter, is the first detected and to date the best characterized of the family of ABC drug efflux transporters. It gained worldwide attention about three decades ago for its role in the phenomenon of multidrug resistance in tumor cells $(8,9)$. Subsequently, constitutive expression of Pgp/MDR1 has been described in a variety of other tissues including liver, intestine, kidney, pancreas, adrenal, capillary endothelium of blood-brain and blood-testis barrier, choroid plexus, placental trophoblast and others (10). The polarized, apical membrane localization of Pgp/MDR1 causes that its substrates are prefeerentially translocated from basolateral to the apical side of the epithelium. Thus, Pgp/ MDR1 limits the influx and facilitates the efflux of its substrates, eventually preventing their intracellular accumulation. Many in vitro and in vivo studies demonstrated high

Address for correspondence:

Hodorova Ingrid, MD, PhD, Department of Anatomy, P.J. Safarik University, Medical Faculty

Srobarova Str. N. 2, 04001 Kosice, Slovak Republic, Phone: ++ 421055 6228866; e-mail: ingrid.hodorova@upjs.sk 
impact of Pgp/MDR1 on drug pharmacokinetics in these organs (10). It is likely that Pgp/MDR1 and other ABCs have evolved in these "normal" tissues to protect them from potentially damaging effect of toxic compounds.

In the kidney, Pgp/MDR1 is expressed mainly at the apical (luminal) brush-border membrane of proximal tubular cells (11) and to lesser extent in the thick limb of Henle's loop, collecting ducts, and glomerular mesangium. Many substances are actively transported by Pgp/MDR1, e.g. calcium channel blockers, immunosuppressants, cardioactive glycosides, antibiotics, antineoplastic and severel others peptides and steroids $(12,13)$. Thus Pgp/MDR1 functions as an efflux pump which represents a protective mechanism to exclude endogenous and exogenous toxins from normal cells and to ultimately excrete them into the bodily secretions. In humans, two genes (MDR1 and MDR2) encode this protein, whereas in rodents there are three homologs of this protein, mdr1 (mdr1b), mdr2, mdr3 (mdr1a) $(14,15,16)$.

The aim of the present study was to determine postnatal expression of Pgp/MDR1 in rat kidney by two widely used monoclonal antibodies for Pgp/MDR1: C219 and UIC2 .These antibodies are known to detect Pgp/MDR1 in paraffin-embedded histological tissue in many histopathological laboratories. The neonatal rat is a useful model for kidney developmental studies since rats are born with immature kidneys and there is considerable postnatal renal development.

\section{MATERIAL AND METHODS}

\section{Animals}

All procedures performed with animals adhered to the permission of the Committee for Ethical Control of Animal Experiments at Safarik University and the permission of the State Veterinary and Food Administration of the Slovak Republic (permission No. 7881/04220/3). All efforts were made to minimize both the number animals and their suffering.

To acquire rat cubs, female Wistar rats were mated with males of the same strain overnight. Tissue from Wistar rat were isolated on the 1st day (D1), 7th day (D7), 14th day (D14), 21st day of life (D21) and from adult animals (60 days old; Ad) by ether anaesthesia. Three animals were killed on each day examined. The kidneys were obtained by medial laparotomy carried out in deeply anaesthetised animals. At the end of surgery rats were killed by overdose of ether. Rat kidneys were immersed in a neutral buffered formalin solution for 72 hours at room temperature. Tissue blocks were dehydrated in increasing alcohols and embedded in paraffin. Seven-micron-thick serial sections were cut and attached to the slide with alum gelatine. The slides were processed for immunohistochemistry.

\section{Antibodies:}

We have used the following primary monoclonal antibodies: mouse anti-MDR1, clone C219 (Signet Laboratories, Inc.) and mouse anti-MDR1, UIC2 - clone C494 (Santa Cruz Biotechnology, Inc.).

\section{Immunohistochemical detection of Pgp:}

For immunohistochemistry, paraffin embedded sections of the rat kidney were deparaffinized with xylene and rehydrated in decreasing ethanols to water. The slides were finally washed in phosphate-buffered saline containing $0.05 \%$ Tween-20 (PBS-Tw), pH 7.6. Endogenous peroxidase activity was blocked by $0.3 \% \mathrm{H} 202$ in methanol for 30 minutes at room temperature. To re-establish an original conformation of epitopes modified after fixation, we performed antigen retrieval using a microwave (in sodium citrate solution for $2 \times 5$ minutes at 500 watts). Pgp/ MDR1 staining procedure continued by blocking nonspecific staining with milk buffer ( $5 \%$ dry milk in TRIS buffer) for 30 minutes at room temperature. Primary antibodies were applied overnight in humidified chamber at $4^{\circ} \mathrm{C}$. After rinsing in PBS-Tw (3x5 minutes) the sections were subsequently incubated with the secondary antibody (30 minutes) and strepatavidin- 
biotin (30 minutes) (Universal detection kit LSAB+KIT/HRP, Dako). Next, the sections were visualized with DAB (3.3'- diaminobenzidine tetrahydrochloride) at a concentration of $0.5 \mathrm{mg}$ / $\mathrm{ml}$ in Tris buffer, $\mathrm{pH} 7.6$ and $0.015 \% \mathrm{H} 2 \mathrm{O} 2$. Slides were stream-rinsed with tap water, counterstained with Mayer's hematoxylin for 2 minutes, washed in tap water, dried, mounted and coverslipped. Sections processed with omission of primary antibody served as control.

\section{Semiquantitative evaluation}

Immunostaining was assessed by two independent observers blinded to animal characteristics.

Expression of Pgp/MDR1 was evaluated separately using the following scale: $3^{+}=$ high level (91-100 \% of positive cells), $2+=$ medium level (11-90 \% of positive cells), $1+=$ low level (up to $10 \%$ of positive cells), - = negative cells ( $0 \%$ of positive cells). Samples with high [3+] and medium level [2+] of proteins expression were considered as positive. Samples scored as $[1+]$ and $[-]$ were considered as negative.

\section{RESULTS}

\section{Kidney of rat cubs}

Using monoclonal antibody mouse anti-MDR1 clone C219 we have detected moderate immunopositivity for Pgp/MDR1 in all developmental stages (D1, D7, D14, D21). Employing semiquantitative evaluation of tissue samples we have found no differences in expression of Pgp/MDR1 in epithelial cells of proximal tubules. The remaining structures (epithelial cells of distal tubules, glomerulus and glomerular capsule) did not show any positivity for Pgp/MDR1.

Using monoclonal antibody mouse anti-MDR1 UIC2 - clone C494 we have detected in all developmental stages the same spatial protein distribution but its signal was significantly weaker.

\section{Kidney of rat adults}

Using both monoclonal antibodies (mouse anti-MDR1:clone C219 and mouse antiMDR1:UIC2 - clone C494) we have observed expression of Pgp/MDR1 in epithelial cells of proximal tubules in adult kidney, too. Employing semiquantitative evaluation we have found no differences in expression of Pgp/MDR1 in all tissue samples. The immunoreactivity for this protein was strongly restricted to the apical membrane and weakly to the cytoplasm of proximal tubular cells. No signal for this protein in the other cells of rat kidney was found.

$\mathbf{A}$

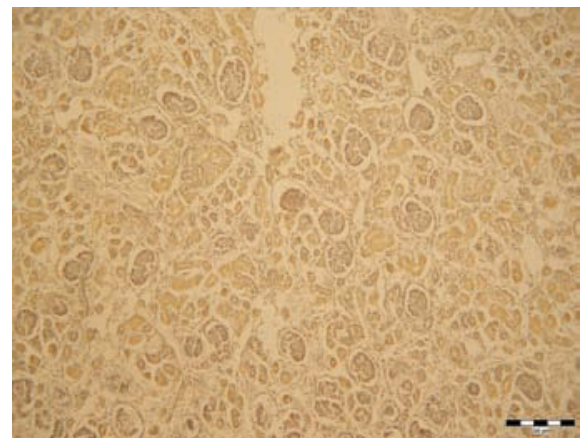

B

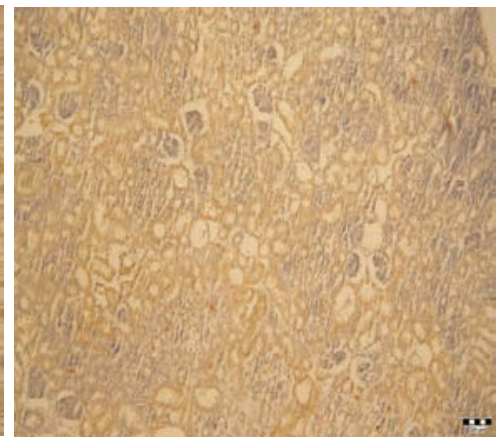

Fig. 1. 1-day old rat kidney - D1. Immunohistochemical detection of Pgp/MDR1 by monoclonal antibody: mouse anti-MDR1 - clone C219 (A), UIC2 - clone C494 (B). Positive expression of Pgp/MDR1 in proximal tubular epithelial cells of rat kidney - cytoplasmic staining (A, B). 

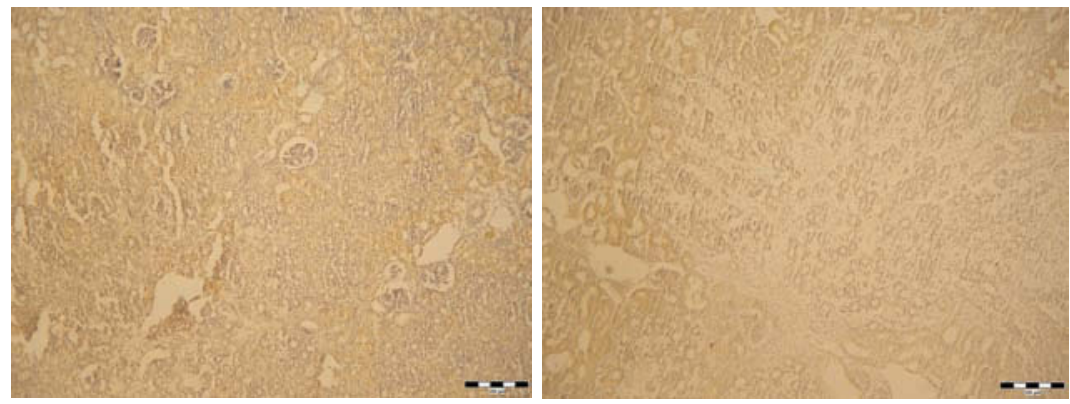

Fig. 2. Pgp/MDR1 was expressed in epithelial cells of proximal tubules in 7-day old rat kidney - D7 (A) and adult kidney (B) by mononoclonal antibody: mouse anti-MDR1 - clone C219. The remaining structures (epithelial cells of distal tubules, glomerulus and glomerular capsule) did not show any positivity for Pgp/MDR1.

\section{DISCUSSION}

Knowledge of the ontogeny about transport proteins involved in distribution and elimination of drugs is important for adequate interpretation of the results of safety studies in juvenile animals. Since rat is one of the most frequently species used in non-clinical testing we used this species in our study on the age-dependent changes in Pgp/MDR1 expression. We focused on the age shortly after birth until early adulthood of the animals (D1, D7, D14, D21, Ad). The transport proteins in the kidney are involved in the passage of compound through the tubular epithelial cells, prior to urinary excretion. Urinary excretion of drugs is a complex interplay of three mechanisms: glomerular filtration, tubular secretion, and tubular reabsorption. The basic prerequisite for excretion into urine is water solubility of drug and/or its metabolites as no transporting carrier is available in this body fluid. Accordingly, the primary mechanism for drug excretion is glomerular filtration, a passive process influenced by molecule size, concentration of unbound fraction of drug in plasma and renal blood perfusion. Tubular secretion and partly also reabsorption are active mechanisms requiring transporters. The function of renal proximal tubular epithelial cells is the most significant factor for active drug renal excretion (17).

The large differences in ontogeny of the individual transport proteins were observed, with some transporters having the highest expression at birth (MRP1 and MRP3), and other having a more or less constant expression during development (MRP2 and BCRP) (18). It was reported that Pgp/MDR expression increased up to Day 26, with a subsequent decrease to adult levels at Day 42 (18). On the other hand, other authors found continuously increasing level of Pgp/MDR1 up to Day 60 (19). In our study we have found relatively constant levels of Pgp/MDR1 protein from the birth to the adulthood.

It should be noted that all our data on transport protein is limited to actual functionality of Pgp/MDR1. The mRNA expression of this transporter was not studied such as in previous mentioned publications. The reason for the differences between the data obtained in our study and those already published, as well as the differences between individual publications, are most likely related to differences in methodology (sample preparation, differences in diet, etc.).

Despite these differences, the results indicate that expression of majority of the transport proteins studied vary during development. Cellular and tissue distribution as well as elimination of transported substrates will therefore also change during development. Knowledge of these ontogenic differences in absorption, distribution, metabolism and excretion (ADME) processes of test compound in development may help to interpret differences in toxicity observed between adult, neonate and young animals. 


\section{REFERENCES}

1. Ambudkar SV, Dey S, Hrycyna CA, Ramachandra M, Pastan I, Gottesman MM. Biochemical, cellular, and pharmacological aspects of the multidrug transporter. Annu Rev Pharmacol Toxicol. 1999; 39:361-98. Review.

2. Stavrovskaya AA. Cellular mechanisms of multidrug resistance of tumor cells. Biochemistry (Mosc). 2000; 65(1):95-106. Review.

3. Higgins C. F. Multiple molecular mechanisms for multidrug resistance transporters.

4. $\quad$ Nature 2007; 446, 749-757.

5. Dean M, Rzhetsky A, Allikmets R. The human ATP-binding cassette (ABC) transporter superfamily. Genome Res. 2001; 11(7):1156-66. Review.

6. Neyfakh AA. Mystery of multidrug transporters: the answer can be simple. Mol Microbiol. 2002; 44(5): 1123-30. Review.

7. Sarkadi B, Homolya L, Szakács G, Váradi A. Human multidrug resistance ABCB and ABCG transporters: participation in a chemoimmunity defense system. Physiol Rev. 2006; 86(4):1179-236. Review.

8. Stavrovskaya AA, Stromskaya TP. Transport proteins of the ABC family and multidrug resistance of tumor cells. Biochemistry (Mosc). 2008; 73(5):592-604. Review.

9. Bosch I, Croop J. P-glycoprotein multidrug resistance and cancer. Biochim Biophys Acta. 1996; 1288(2):F37-54. Review.

10. Goldstein LJ, Gottesman MM, Pastan I. Expression of the MDR1 gene in human cancers. Cancer Treat Res. 1991; 57:101-119. Review.

11. Schinkel AH, Jonker JW. Mammalian drug efflux transporters of the ATP binding cassette (ABC) family: an overview. Adv Drug Deliv Rev. 2003; 55(1):3-29. Review.

12. Thiebaut F, Tsuruo T, Hamada H, Gottesman MM, Pastan I, Willingham MC. Cellular localization of the multidrug-resistance gene product P-glycoprotein in normal human tissues. Proc Natl Acad Sci U S A. 1987; 84(21):7735-7738.

13. Ernest S, Bello-reuss E. P-glycoprotein functions and substrates: possible roles of MDR1 gene in the kidney. Kidney Int 1998; 65: S11-S17.

14. Shinkel AH. The physiological function of drug-transporting P-glycoproteins. Semin Cancer Biol 1997; 8:161-170.

15. Eraly SA, Blantz RC, Bhatnagar V, Nigam SK. Novel SK. Novel aspects of renal organic anion and cation transporters. Curr Opin Nephrol Hypertens 2003; 12: 551-558.

16. Gottesman MM and Pastan I. Biochemistry of multidrug resistance mediated by multidrug transporter. Annu Rev Biochem 1993; 62: 385-427.

17. Inui KI, Masuda S, Saito H. Cellular and molecular aspects of drug transport in the kidney. Kidney Int 2000; 58: 944-958.

18. Lash LH, Putt DA, Cai H. Drug metabolism enzyme expression and activity in primary cultures of human proximal tubular cells. Toxicology 2008, 244:56-65.

19. de Zwart L, Scholten M, Monbaliu JG, Annaert PP, Van Houdt JM, Van den Wyngaert I, De Schaepdrijver LM, Bailey GP, Coogan TP, Coussement WC, Mannens GS. .The ontogeny of drug metabolizing enzymes and transporters in the rat. Reprod Toxicol. 2008, 26(3-4):220-230.

20. Rosati A, Maniori S, Decorti G, Candussio L, Giraldi T, Bartoli F. Physiological regulation of P-glycoprotein, MRP1, MRP2 and cytochrome P450 3A2 during rat ontogeny. Dev Growth Differ. 2003 Aug;45(4):377-387.

Acknowledgement: This work was supported partly by grant VEGA 1/0388/08 and partly by grant VVGS 25/10-11.

Received: November,12,2010

Accepted: December,16,2010 